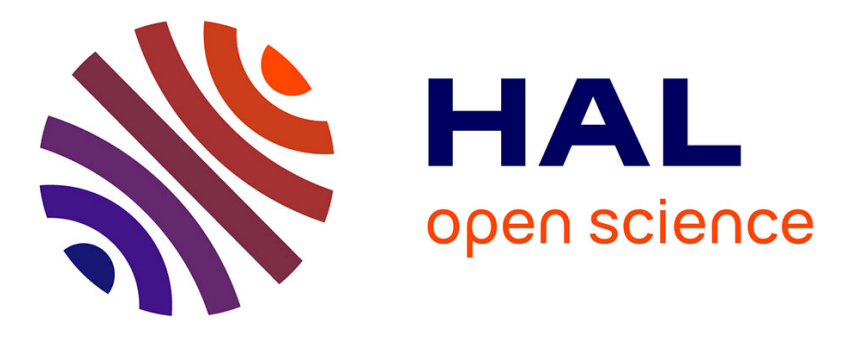

\title{
All-Dielectric Color Filters Using SiGe-Based Mie Resonator Arrays
}

\author{
Thomas Wood, Meher Naffouti, Johann Berthelot, Thomas David, \\ Jean-Benoît Claude, Léo Métayer, Anne Delobbe, Luc Favre, Antoine Ronda, \\ Isabelle Berbezier, et al.
}

\section{To cite this version:}

Thomas Wood, Meher Naffouti, Johann Berthelot, Thomas David, Jean-Benoît Claude, et al.. AllDielectric Color Filters Using SiGe-Based Mie Resonator Arrays. ACS photonics, 2017, 4 (4), pp.873 - 883. 10.1021/acsphotonics.6b00944 . hal-01788638

\section{HAL Id: hal-01788638 https://hal.science/hal-01788638}

Submitted on 22 May 2018

HAL is a multi-disciplinary open access archive for the deposit and dissemination of scientific research documents, whether they are published or not. The documents may come from teaching and research institutions in France or abroad, or from public or private research centers.
L'archive ouverte pluridisciplinaire HAL, est destinée au dépôt et à la diffusion de documents scientifiques de niveau recherche, publiés ou non, émanant des établissements d'enseignement et de recherche français ou étrangers, des laboratoires publics ou privés. 


\title{
All-Dielectric colour filters using SiGe-based Mie resonator arrays
}

\author{
Thomas Wood ${ }^{\mathrm{a}, *, \dagger}$, Meher Naffouti ${ }^{\mathrm{a}, \mathrm{b},+}$, Johann Berthelot ${ }^{\mathrm{c}}$, Thomas David ${ }^{\mathrm{a}}$, Jean-Benoît Claude ${ }^{\mathrm{a}}$ \\ Léo Métayer ${ }^{\mathrm{a}}$, Anne Delobbe ${ }^{\mathrm{d}}$, Luc Favre ${ }^{\mathrm{a}}$, Antoine Ronda ${ }^{\mathrm{a}}$, Isabelle Berbezier ${ }^{\mathrm{a}}$, Nicolas Bonod ${ }^{\mathrm{c}}$ \\ and Marco Abbarchi ${ }^{\mathrm{a}, * *}$ \\ ${ }^{a}$ CNRS, Aix-Marseille Université, Centrale Marseille, IM2NP, UMR 7334, Campus de St. Jérôme, 13397 Marseille, \\ France \\ bLaboratoire de Micro-Optoélectroniques et Nanostructures, Faculté des Sciences de Monastir, Université de \\ Monastir, Monastir 5019, Tunisia \\ cAix-Marseille Univ, CNRS, Centrale Marseille, Institut Fresnel, Marseille, France \\ ${ }^{d}$ Orsay Physics 13710 Fuveau, France
}

KEYWORDS : SiGe alloys, Mie resonator, solid-state dewetting, colour-filters, Finite Difference - Time Domain simulations, surface functionalisation.

\begin{abstract}
Dielectric Mie resonators have attracted a great deal of attention over the past few years thanks to their remarkable capabilities in manipulating light propagation at the nanoscale. However, the practical implementation of technological products is still elusive. One of the important limits is the absence of a high-performing material and a fabrication method that can be easily integrated into modern micro-electronic devices at affordable costs. Here, we provide theoretical and experimental evidence of an alternative semiconductor material, SiGe alloys, for dielectric Mie resonator applications. As a material compatible with the processing requirements of the semiconductor industry, it possesses comparable optical properties to its conventional Si-based counterpart at visible frequencies in spite of its higher optical losses. These dielectric resonant particles can be obtained over very large surfaces on arbitrary silica substrates via spontaneous solid state dewetting of ultra-thin $(<1$ oonm) SiGe amorphous layers. Furthermore, the polycrystalline SiGe islands can be precisely organised in uniform arrays featuring low size dispersion. As an example, we demonstrate SiGebased Mie resonator arrays functioning as colour pass-band filters across the full visible spectral range. The filters function both in transmission and diffusion and are fabricated using a methodology compatible with C-MOS fabrication.
\end{abstract}

Introduction

Light management using dielectric Mie resonators (MRs)', particularly Silicon nano-particles, has been the subject of intense interest in the literature for the past decade $\mathrm{d}^{2,3,4}$. This work has led to a host of innovative applications in domains such as sensing and photovoltaics where efficient light trapping is required $5,6,7,8$. Of particular note in this area is the substrate-coupling phenomenon, whereby MRs are able to channel optical energy from the ambient towards a bulk material by the extension of the resonantly confined fields into the substrate, as this mechanism forms the crux of the required "trapping" behaviour ${ }^{9,10}$. Furthermore, modifying geometric properties of the MRs allows for control over the light-matter interaction, beginning with the well-known interplay between the resonant wavelengths and the size and refractive index of the $\mathrm{MRs}^{11,12}$. More complex examples include the coupling of two or more resonators allowing for polarisationsensitive interactions ${ }^{13,14}$ and controlling the morphology of the MRs to determine the angular selectivity of the soformed metasurfaces ${ }^{15,16}$. We note also that due to their low loss at visible and NIR optical frequencies, all-dielectric Mie resonators are fast surpassing plasmonics as the dominant technique for managing light-matter interactions at sub-wavelength scales.

Over the last decade, during which MRs have been studied intensively from both a theoretical and practical point of view, fabrication methods have been refined to allow for the formation of arrays of precisely sized and positioned resonant particles. Techniques including e-beam lithography coupled with reactive ion etching ${ }^{17}$ or chemical etching $^{18}$, ion sputtering ${ }^{19}$, nano-imprinting ${ }^{20}$ and laserbased writing ${ }^{21}$ and ablation ${ }^{22}$ methods have the advantage of producing MR arrays featuring islands with low size dispersions and well-defined positions on their substrates. 
Another emerging method for the fabrication of MRs is solid state dewetting of monocrystalline $\mathrm{SOI}^{23,24,25,26}$ type structures, consisting of ultra-thin ( $10 \mathrm{~nm})$ Si layers on a buried oxide (BOX) layer atop a silicon substrate. This method is suitable for application to large surfaces, at the expense of sacrificing the spatial organisation and fine control over the islands' dimensions ${ }^{27,28}$ and relative positions. The technique can be refined through the addition of a templating step ${ }^{29}$, whereby narrow $(<100 n m)$ trenches are milled before the dewetting (e.g. by direct etching via focused ion beam, FIB), providing excellent control over the spatial positioning and size distribution of the formed MRs.

So far, silicon has been the material of choice for the implementation of this class of resonant dielectric particles and related meta-surfaces. Other materials of note are gallium phosphide $(\mathrm{GaP})^{30}$, aluminium gallium arsenide $(\mathrm{AlGaAs})^{31}$ and titania $\left(\mathrm{TiO}_{2}\right)^{20,32,33,34,35}$. To date, no reports have been shown in this field on silicon germanium ( $\mathrm{SiGe}$ ) alloys, despite their clear advantage of exhibiting larger refractive indices with respect to pure $\mathrm{Si}^{36,37}$ allowing, in principle, for better light confinement. We note that, due to its high absorption in the visible region ${ }^{6}$ that prohibits the formation of high quality resonances, germanium alone is not a desirable material for MR fabrication. Nevertheless, the use of Ge with $\mathrm{Si}$ for dewetting provides a strategic tool for controlling the dynamics of islands formation: it allows for tuning the island densities (e.g. higher $^{38}$ for the case of spontaneous dewetting, or lower ${ }^{29}$ when using templating for guiding the dewetting fronts), speeds-up the self-assembly allowing for a reduced processing temperature and overall dewetting time and controls the islands' shape by increasing the vertical aspect ratio. In contrast with more conventional fabrication approaches (e.g. e-beam lithography and reactive ion etching) where only cylinders can be obtained, our hybrid top-down/bottom-up method of "templated dewetting" of $\mathrm{SiGe}$ enables a true $3 \mathrm{D}$ shaping of the islands. Furthermore, it has recently been shown that the use of SiGe for dewetting can lead to complex arrangements (e.g. bimodal size distributions) of precisely positioned islands; a clear first step to more complex optical functions and more "striking" results with Mie resonator oligomers ${ }^{29}$. Furthermore, the use of SiGe coupled with $\mathrm{Ge}$ condensation has allowed for the implementation of coreshell structures integrated in the dewetted islands for the first time ${ }^{38}$. Very importantly, the integration of SiGebased hetero-structures within dewetted islands may lead to the implementation of light emitters in the resonators. This elusive point in the community of Mie resonators has been recently addressed for NIR frequencies emission with pure Ge antennas ${ }^{39,40}$ confirming the potential of this approach with respect to other dielectric non-emitting materials.

From an optical point of view, the imaginary component of the refractive index of pure $\mathrm{Ge}$ is orders of magnitude higher than for pure Si over a large part of the visible spectral region (see Supporting Information), explaining the need to minimise the concentration of Ge in SiGe alloys if nanostructures displaying low absorption at short visible wavelengths are to be obtained. This constraint is less important at near-infrared frequencies where $\mathrm{Ge}$ absorption is reduced. This observation opens the opportunity to use larger Ge concentrations exploiting a larger refractive index enhancing the light confinement and obtaining sharper resonances ${ }^{21}$.

Solid state dewetting has been extended to amorphous silicon on arbitrary silica substrates ${ }^{24}$ thus relaxing the requirement to use commercial monocrystalline SOIs and allowing for a precise tuning of the buried oxide (BOX) thickness, which has been shown to strongly influence the resonant wavelengths of $\mathrm{MRs}^{3}$. Furthermore, using amorphous layers allows one to functionalise a full substrate at temperatures under $600^{\circ} \mathrm{C}$ for Si-based resonators $4^{4,42}$ and lower than $300^{\circ} \mathrm{C}$ for Ge-based systems in only 30 minutes ${ }^{43}$. An intermediate temperature and comparable timescale are therefore expected for SiGe alloys.

This work centres on the use of arrays of resonant particles formed by templated dewetting of SiGe films in order to produce wavelength-tuneable visible domain colour filters working in reflection/transmission as well as in diffusion. The substrate-coupling phenomenon is refined with a semi-isolating oxide layer of an optimised thickness to create filters displaying narrow, tuneable pass-bands. The association of thermal dewetting with a minimised templating step allows us to combine the advantages of both techniques: large surface treatment for the former, and precise control of particle size and position for the latter. Care has been taken to address the integration of the filters with existing fabrication constraints on semiconductor imaging devices. To this end, the structures are all-dielectric (i.e. no metallic inclusions that could disrupt the charge transport in opto-electronic devices) and feature an optically- and electrically-isolating $\mathrm{SiO}_{2}$ layer. We demonstrate the feasibility of our technique for the fabrication of practical filters by fabricating arrays with varying pass bands with dimensions of $15 \times 15 \mu \mathrm{m}$, similar to the pixel size commonly used in CMOS/CCD imaging devices.

\section{Simulations}

The spectral position and efficiency of pass-band MRbased filters depend on a large number of coupled parameters, including the refractive index of the materials, resonator size and shape, array periodicity, isolation with respect to the substrate, and more. The simulations to determine the optimal parameters for our band-pass filters were performed on a home-made FDTD code written in $\mathrm{C}++$. We chose to restrict the lateral (in-substrate-plane) arrangement of MRs to a square lattice. The refractive indices used in the simulations were $\mathrm{n}_{\mathrm{Si} / \mathrm{SiGe,sim}}=4, \mathrm{n}_{\mathrm{SiO} 2, \mathrm{sim}}=$

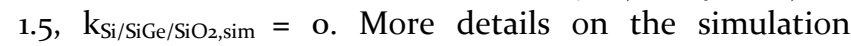
methods can be found in the Supporting Information. 
Initial simulations were focused on determining the required aspect ratio for the MRs, defined as $\eta=h / r$, assuming an ellipsoid structure. Resonators with a low aspect ratio couple strongly the incident light with the substrate due to their high cross-section with the substrate with respect to their overall volume ${ }^{2}$; they give rise to wide, low contrast pass-bands unsuitable for our purpose. Increasing the aspect ratio of the resonators instead, allows for better defined pass-bands, with acceptable performances for $\eta^{\prime} \mathbf{1}^{3}$. This is the main reason for using small amounts of Ge in the alloy: while pure Si dewetting leads to a limited $\eta$ (see for instance reference [27]), a small Ge content transforms the shape into hemispheres (see references $[47,48])$, providing a more suitable shape $(\eta \sim 1)$ for achieving sharper photonic resonances.

Next, we investigate the effect of the array periodicity on the filter performances. Fig. 1 (a) shows the simulated reflectance for an array of hemispherical MRs of radius 9onm for various periodicities $(\mathrm{P})$ atop $150 \mathrm{~nm} \mathrm{SiO}_{2}$. For low values of $\mathrm{P}(<350 \mathrm{~nm})$, low FWHM (<100nm) peaks approaching $100 \%$ reflection efficiency can be seen in the reflectance spectra around 50onm, superimposed on a low reflectance background. Although such characteristics are relevant to a special class of filter featuring an "inversed" pass-band (wherein light at all but a narrow wavelength range is efficiently coupled to the substrate), fabrication constraints have to-date prevented the realisation of such structures via solid state dewetting. As the periodicity of the array increases, the reflectance peak reduces in amplitude, eventually giving way to a less pronounced dip for $\mathrm{P}>4$ oonm. The degradation of the filtering properties on increasing $\mathrm{P}$ is to be expected, as the portion of the surface area covered by the resonators, linked to the lightmatter interaction cross-section, is reduced.
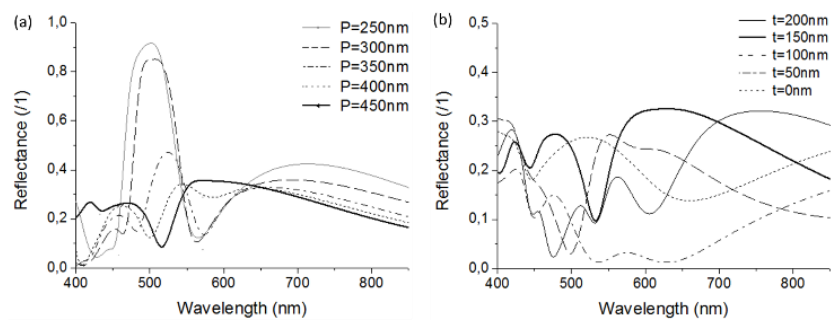

Fig. 1 Simulated reflectance spectra for (a) various lateral periodicities $\mathrm{P}$ for hemispherical resonators of radius 9onm on a $\mathrm{Si}$ substrate topped with 150 mm of $\mathrm{SiO}_{2}$. (b) Various $\mathrm{SiO}_{2}$ layer thicknesses $t$ for hemispherical resonators of radius $90 \mathrm{~nm}$ in an array of periodicity $450 \mathrm{~nm}$.

A further parameter to be considered is the thickness $t$ of the BOX layer separating the MRs from the substrate. It has already been shown in the literature ${ }^{2,3}$ that, if the oxide thickness is of the order of the wavelength of incoming light, this layer can act as a Fabry-Perot cavity whereby it effectively determines the wavelengths available to undergo resonance in the MRs through constructive or destructive interference phenomena upon reflection. Furthermore, if this layer is too thick then light at resonant wavelengths is poorly coupled to the substrate, whilst if it is too thin resonances are broadened and their contrast decreased (see Fig. 1 (b)). From the analysis performed for an array of hemispheres of radius $r=90 n m$ with $a$ periodicity $\mathrm{P}=450 \mathrm{~nm}$ a single pronounced dip in reflectance is observed for $\mathrm{t}=100$ and slightly red-shifted, at $t=150$ mm. These two values therefore constitute the bounds of the acceptable range of $t$.
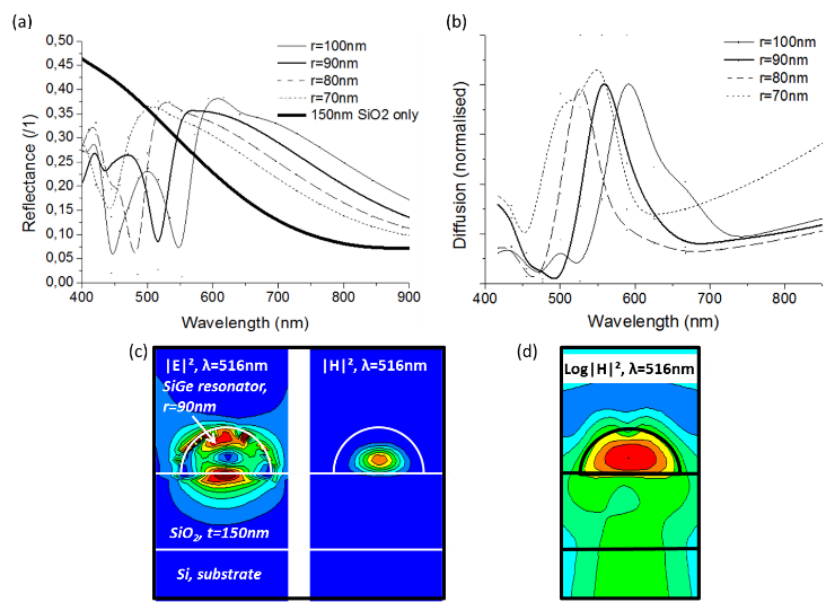

Fig. 2 (a) Reflectance and (b) diffusion spectra for hemispherical resonators with various radii $r$ in an array of $450 \mathrm{~nm}$ periodicity on a $\mathrm{Si}$ substrate topped with $150 \mathrm{~nm}$ of $\mathrm{SiO}_{2}$. Also shown for comparison is the simulated spectral response of a $\mathrm{Si}$ substrate coated with the same $\mathrm{SiO}_{2}$ thickness without resonators. (c) Electric and magnetic near-field profiles at the reflectance dip at $\lambda=516 \mathrm{~nm}$ for hemispheres of radius $r=90 n m$. (d) Logarithm of near-magnetic field profile shown in (c).

Clearly the most critical parameter to be set is the resonator's optical volume, by which we mean the product of the physical volume and the material refractive index, as this determines the wavelengths of the Mie resonances. Naturally, a greater volume yields resonances that are redshifted with respect to those of smaller resonators, together with permitting higher order modes (e.g. electric and magnetic quadrupoles ${ }^{2}$. For a hemispherical resonator, the volume can be simply translated to the radius of the particle. Fig. 2 (a) shows the simulated reflectance and Fig. 2 (b) the simulated diffusion spectra of hemispherical resonators with varying radii in arrays of periodicity $\mathrm{P}=450 \mathrm{~nm}$ on a $\mathrm{Si}$ substrate with an oxide thickness $t=150$ mm. A common reflectance dip occurs at 45onm, equal to the array periodicity, corresponding to the Rayleigh anomaly where the first diffracted order in the air above the system ceases to exist. This dip is more pronounced for resonators with larger radii, as the diffraction efficiency of the $2 \mathrm{D}$ grating that the array constitutes is increased. The centre wavelength of the reflectance dip corresponding to the Mie resonance mediated pass-band is clearly seen to red-shift upon increasing the particle radius, passing from $\lambda=450 \mathrm{~nm}$ for $\mathrm{r}=70 \mathrm{~nm}$ (where the reflectance dip is superimposed on the Rayleigh anomaly), to $\lambda=550 \mathrm{~nm}$ for $r=100 \mathrm{~nm}$. We see a similar red-shift of the diffusion peaks in Fig. 2 (b). 
Further to the fact that the pass-bands are seen to occur near the resonant wavelengths, the FDTD simulations allow us to gain an insight into the phenomena giving rise to their formation. Fig. 2 (c) shows the near field distributions of the electric and magnetic fields at the wavelength corresponding to the reflectance minimum for $\mathrm{r}=90 \mathrm{~nm}$ in Fig. 2 (a): a magnetic field node is centred in the hemispherical resonator encircled by an electric displacement current. This confirms that the fundamental Mie resonances are indeed responsible for the features observed in the simulated spectra. Upon plotting the logarithm of the magnetic field distribution (Fig. 2 (d)), it is clearly seen that the field associated with the resonance also extends through the BOX layer into the Si substrate, constituting the substrate-coupling phenomenon which provides the selective transmittance needed to tune the filter pass-bands. In order to demonstrate the efficiency of the combined BOX-resonator array structure on modulating the reflectance spectra, Fig. 2 (a) also shows the simulated response of a $\mathrm{Si}$ substrate coated only with $150 n m \mathrm{SiO}_{2}$. It can be seen that no rapid modulation of the reflectance, needed to create spectrally narrow filters, is observed without the additional presence of the Mie resonator arrays.

To summarise, we can conclude from the simulations that the optimal geometry for the SiGe-based MRs filters is: hemispherical islands with radii varying between roughly 70 and $150 n m$ atop $100-150 \mathrm{om}$ of $\mathrm{SiO}_{2}$ on a silicon substrate.

\section{Experimental}

The outcome of the simulations calls for a very unique silicon-germanium on insulator (SGOI) structure, particularly in terms of the $\mathrm{SiO}_{2} \mathrm{BOX}$ thickness, which is not commercially available. We have therefore resorted to fabricating our own custom SGOI structures. As mentioned earlier, in order to obtain MRs with an aspect ratio close to 1, we have added Ge to the Si thin film on top of the BOX. We limit the percentage of Ge to around $20 \%$ $\left(\mathrm{Si}_{0.8} \mathrm{Ge}_{0.2}\right)$ in order to yield hemispherical islands whilst avoiding strong optical absorption in the visible spectral region $44,45,46$. Such concentrations are, however, large enough to induce dramatic modifications of the dewetting dynamics and of the equilibrium shape of SiGe islands 47,48 : a vertical aspect ratio of $\sim 1$ and improved in-plane symmetry can be obtained via Ge alloying during dewetting in contrast with pure Si featuring very flat islands.

Two samples were fabricated, named A and B, with thicknesses of zonm and 5onm for the initial flat SiGe layer respectively. As detailed later, these thicknesses allow us to achieve MRs of the dimensions required with a relatively low periodicity that is typically below the pass-band wavelength. For both samples the BOX thickness was 150 $\mathrm{nm}$. The key steps of the fabrication process are shown in Fig. 3 (a).
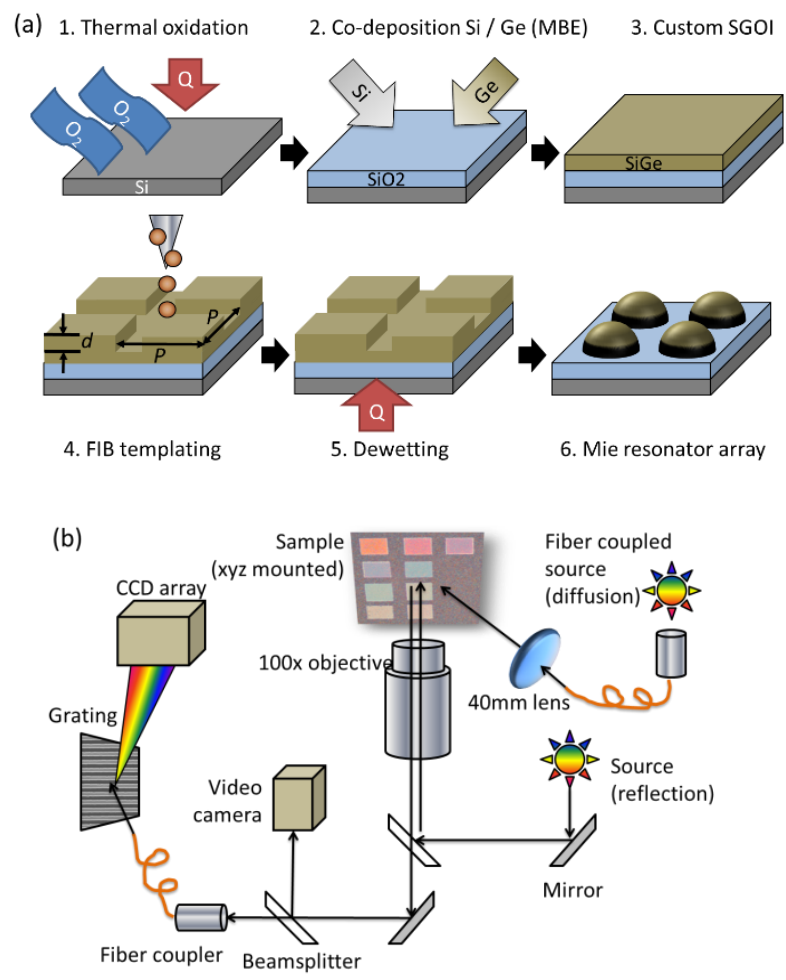

Fig. 3 (a) Diagram showing the steps involved in the fabrication of MR arrays. Panel 4. shows the periodicity P and milling depth $\mathrm{d}$. (b) Spectroscopy bench used for reflectance and diffusion measurements.

The custom SGOI substrates were prepared via a two-step process; firstly, thermal oxidation of crystalline $\mathrm{Si}$ wafers was carried out in a rapid thermal processing oven, followed by deposition of the SiGe alloys under ultra-high vacuum (UHV) in a molecular beam epitaxy (MBE) reactor. The samples were then transferred to a mass-filtered liquid-metal ion source FIB (LMIS-FIB) milling system for templating prior to dewetting. Groups of square lattices approximately $15 \times 15 \mu \mathrm{m}$ in size were milled, featuring periodicities of 250, 290, 330, 360, 400, 450, 500, 550, 6oonm in both spatial directions in order to yield resonators with the sizes determined previously by the simulations (radii ranging from 70 to $150 \mathrm{~nm}$ ). Eight groups of lattices (each with $250 \leq \mathrm{P} \leq 600 \mathrm{~nm}$ ) were milled on each sample, with 4 different nominal target milling depth d: 2 , 4, 6, 7nm. After ion milling the samples were then returned to the UHV chamber of the MBE reactor for dewetting (annealing) at $700^{\circ} \mathrm{C}$ for 3omins. After annealing the dewetted islands are no longer constituted of amorphous material, but are made up of polycrystalline $\mathrm{SiGe}^{28}$. This transition to a polycrystalline phase has been demonstrated in the literature to lead to narrower and intensified resonances ${ }^{21}$.

The samples were initially characterized by bright- and dark-field optical microscopy in order to observe the reflected and diffused colours, as well as to obtain an indication of the homogeneity of the resonator arrays formed. Following this, diffusion and reflection spectra at visible and near infrared frequencies were collected on a 
home-made spectroscopy bench, shown in Fig. 3 (b). The samples were further characterised by atomic force microscopy (AFM) and scanning electron microscopy (SEM).

\section{Optical characterisation results}

The palettes in Fig. 4 show the reflected and diffused colours observed with the imaging optical microscope as a function of the array periodicity $\mathrm{P}$ and the milling depth $\mathrm{d}$ for samples A and B. Patterns sharing the same milling depth are arranged on a same row, with the period increasing from left to right. The effect of these two parameters on the size of the MRs formed after dewetting is best understood through the diffused colours: for a given $\mathrm{d}$, increasing $\mathrm{P}$ yields larger resonators as shown by the diffused colour transitioning from short to long wavelengths (e.g. blue to red for sample A on row $\mathrm{d}=6 \mathrm{~nm}$, from r1 to $\mathrm{r}_{5}$ ), as expected. For the highest periodicities, the diffused colours begin to taint blue due to the MRs supporting multiple resonant modes, as discussed in more detail in the spectroscopic measurement analysis. At a fixed periodicity, increasing the milling depth yields smaller resonators due to the fact that deeper milling sputters more material and the diffused colours blue-shift.

(a) Sample A

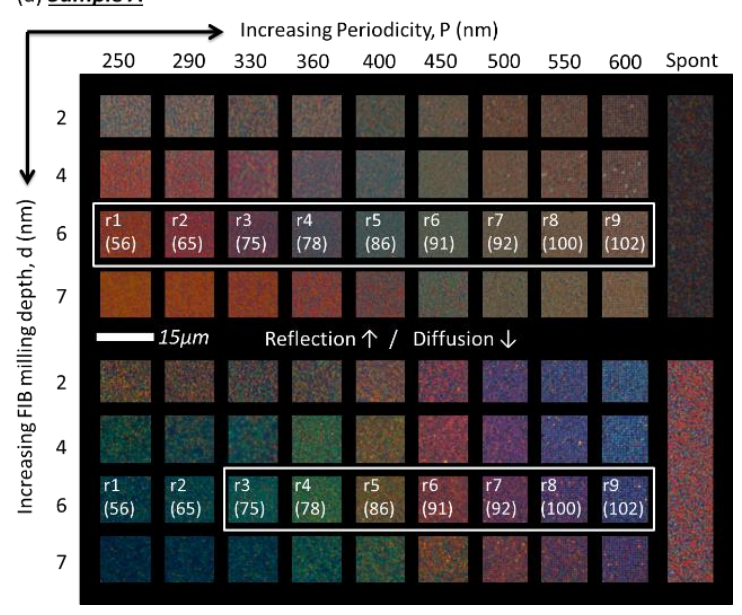

(b) Sample B

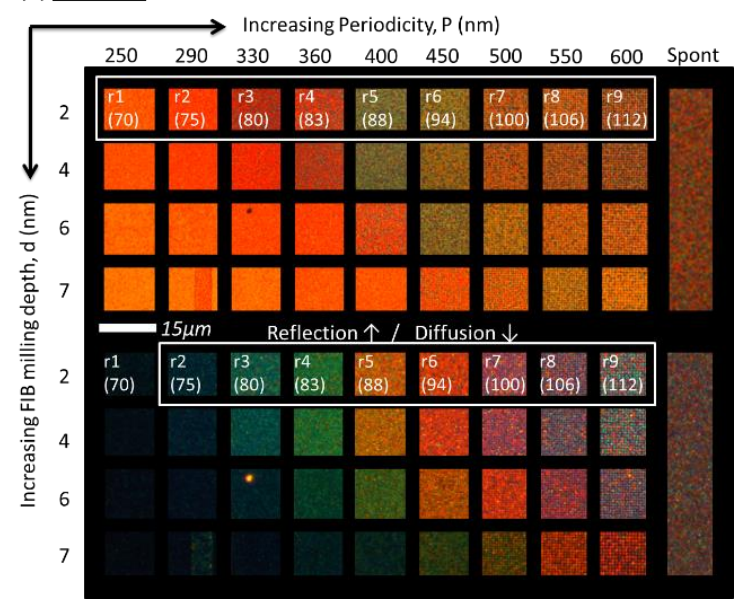

Fig. 4. Palettes formed from bright-field (reflectance) and dark-field (diffusion) optical images of resonator arrays of varying periodicities $\mathrm{P}$ with different templating milling depths $d$ (a) on sample A, (b) on sample B. To the right of each panel (columns labelled "Spont") is an image of the spontaneously dewetted region of each sample viewed in both bright- and dark-field. The numbering of arrays is used in the optical and physical characterisations, whilst arrays in white boxes are those whose CIE chromaticity coefficients feature in Fig. 5 and Fig 6 . The numbers in brackets correspond to the estimated resonator radii in $\mathrm{nm}$, as reported in Fig 7 (c) for sample A. Each $15 \mu \mathrm{m} \times 15 \mu \mathrm{m}$ dewetted pattern is here surrounded by a black cornice in order to better highlight and compare the reflected and scattered colours. An image of the original configuration of the sample is provided in Figure $\mathrm{S}_{3}$ in the Supporting Information only for the specific cases of 6 $\mathrm{nm}$ milling depth of sample A (third line of the palette in (a)) and $2 \mathrm{~nm}$ milling depth for sample B (first line of the palette in (b)). In Fig. S3 (Supporting Information) the spontaneously dewetted areas between the etched patterns are not masked with a black cornice. For the sake of thoroughness, SEM images including patterned and un-patterned areas are displayed in Fig 7 (a).

For the remaining characterisations, we have focussed for sample A on the line of pixels formed at milling depth $\mathrm{d}=6 \mathrm{~nm}$, and for sample $\mathrm{B}$ on the line corresponding to $\mathrm{d}=\mathbf{2 n m}$, since these yield diffused colours covering the entire visible spectral region. These pixels are numbered $\mathrm{r} 1$ - r9 in Fig. 4. Referring to Fig. 5 (a,c) and Fig 6 (a,d), showing the collected diffusion (D) and reflectance (R) spectra for these pixels, the general trends can be summarised as follows: as the resonator size increases, the peaks in the diffusion spectra are seen to shift from blue to red ( $\mathrm{r} 2$ through to $\mathrm{r} 7 / 8$ ), as well as multiplying in number for the highest periodicities ( $\mathrm{r} 6, \mathrm{r} 7 \& \mathrm{r} 8$ ), corresponding to the presence of multiple resonance orders in the islands. In the reflectance spectra, minima can be observed at spectral positions near the diffusion peaks due to the substrate coupling phenomenon described in the simulations section. Either side of these minima, the reflectance spectra are seen to exhibit maxima whose positions determine the apparent reflected colour of the arrays. The trends described previously in the experimental reflectance spectra presented in Fig $6(\mathrm{a}, \mathrm{d})$ are in good qualitative agreement with the simulation results. Further simulations presented in the Supporting Information (taking into account the true sizes of the resonators as measured and presented in the Physical Characterisation results section) allow for a direct comparison between the simulation and experimental results. We note that in order to estimate the transmittance $\mathrm{T}$ of the MR arrays, representing the intensity received by a photo-sensitive structure buried in the substrate and featuring the passbands mentioned previously, the simple expression $\mathrm{T}=1-$ $\mathrm{R}$ can be used (assuming the absorption in the resonators to be negligible). The investigation of $\mathrm{R}$ for both samples in spontaneously dewetted areas (such as those shown on the right of each palette in Fig. 4) is shown in the Supporting Information. Even though this discussion goes beyond the aim of this paper, we note that spontaneous 
dewetting could be exploited for the fabrication of broadband and broad- angle anti-reflection coatings. In fact, disordered systems may outperform their ordered counterparts as has been shown recently ${ }^{49,50}$. A hint of this possibility can be found in the lower reflected intensity of the spontaneously dewetted region in Fig. 4 (a) and quantified by the low reflectivity spectrum shown in the Supporting Information in Fig. $\mathrm{S}_{3}$ (d) (R 10\% over a broad spectral range). Nonetheless, this task would require a large reduction of the BOX thickness underneath the SiGe resonators, as shown by the simulations in Fig. 1 (b). From a fundamental research point of view, it is worth mentioning that disordered arrays of resonators could be extremely appealing for the investigation of light diffusion in complex media ${ }^{51,52,53,54}$.

To complete the optical characterisation of the samples, and to quantify the filters' performances in a standardised way, the chromaticity coefficients have been calculated and placed on the colour space (gamut) defined by the 1931 Commission Internationale de l'Eclairage (CIE) for the pixels indicated by white boxes on the colour palettes in Fig. 4. First, the $\mathrm{X}, \mathrm{Y}, \mathrm{Z}$ chromaticity components are calculated using Eq. 1, and the analogous versions for the $Y$ and $\mathrm{Z}$ components, by integrating the spectral response of the pixels in both reflectance $R(\lambda)$ and diffusion $D(\lambda)$ configurations with the corresponding colour matching functions (CMF) $\bar{x}, \bar{y}, \bar{z}$ shown in Fig. 5 (e). We note here that a "flat" (non-wavelength dependent) source has been used in the calculations, such that the colours plotted represent the un-convoluted performances of the filters. The reduced parameters $\mathrm{x}, \mathrm{y}$ are then calculated using Eqs. 2 and 3.

$$
\begin{array}{lrr}
X=\int R, D(\lambda) \bar{x}(\lambda) d \lambda & x=\frac{X}{X+Y+Z} & y=\frac{Y}{X+Y+Z} \\
\text { Eq1 } & \text { Eq2 }
\end{array}
$$

Fig. 5 (b,d) and Fig 6 (b,e) show the coordinates of selected pixels on both samples operating in diffusion and reflection respectively on the full colour space, whilst Fig 6 $(c, e)$ are zooms on the indicated central regions. In both reflection and diffusion configurations, and for both samples A and B, the plotted coordinates encircle the white-point indicating that a full colour palette is available with the filters. The radial distance from the white-point is lower in the reflection configuration, owing to the high "background" seen in the reflectance spectra from which the pass-band peak is subtracted making the filters less efficient. The purity of the filtered colours is highest for the smallest MRs (i.e. r1 is less "white" than r9), due to the fact that the larger islands support multiple resonant modes ${ }^{2}$. This accounts for the "spiralling-in" towards the whitepoint of the plotted coordinates with increasing MR size. Comparing samples $\mathrm{A}$ and $\mathrm{B}$, it can be seen that the "quality" of the colours obtained with sample B is higher due to the increased radial distance from the white-point that is typically observed.
It is worth stressing that, in spite of a larger extinction coefficient of Ge with respect to $\mathrm{Si}$ at shorter wavelengths (see Supporting Information, Figure S2 (b)) where the dielectric Mie resonators exhibit only the lowest order resonances (the broadest), a relatively high $\mathrm{Q}$ factor can be found: as an example, the case of r2 in Fig. 5 (c) shows a resonance at $460 \mathrm{~nm}$ with a $\mathrm{Q}$ factor of about 13 . This value of $\mathrm{Q}$ for $20 \% \mathrm{SiGe}$ compares well with the values reported for pure $\mathrm{Si}$ at the same frequencies ${ }^{2,55}$. At the same time this measured value of $Q$ clearly outperforms the case of pure Si dewetting (shown reference [27] where the islands featured a low $\eta$ ) accounting for the beneficial effect of an appropriate shape of the resonant particles $(\eta \sim 1)$.

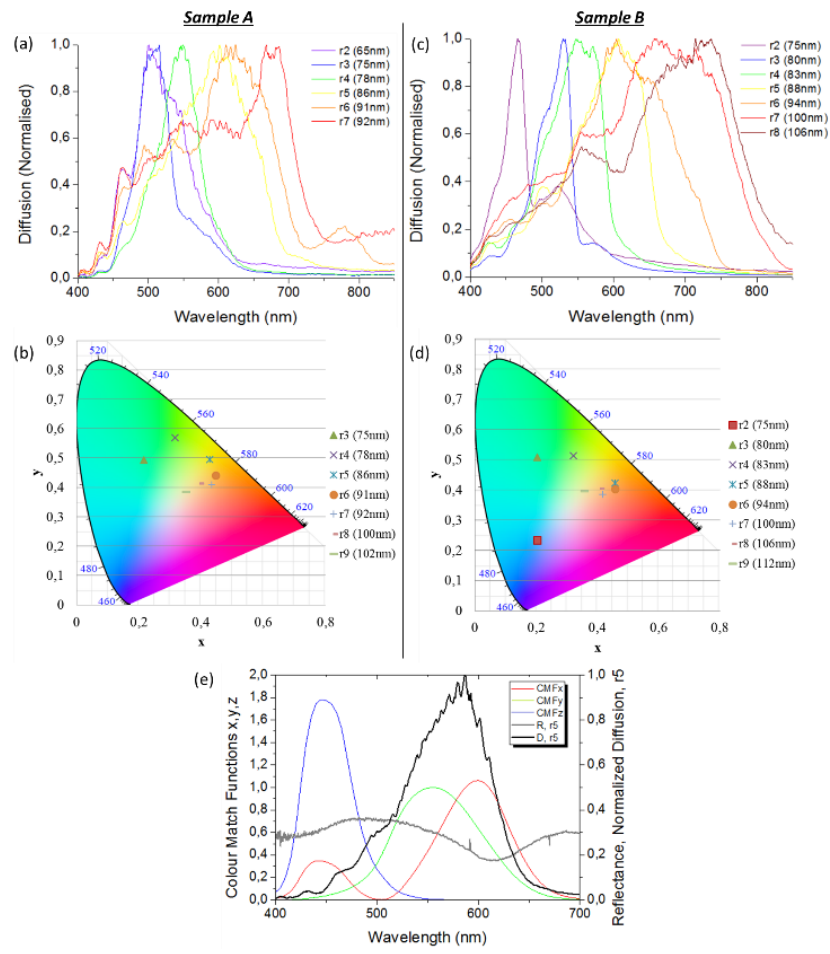

Fig. 5 (a,c) Scattering spectra for selected pixels on samples A and $B$ respectively, each curve is self-normalised. (b,d) CIE chromaticity gamut for selected pixels on samples A and B respectively based on the scattering spectra. The numbers in brackets correspond to the estimated resonator radii in $\mathrm{nm}$. (e) Colour matching functions used to calculate chromaticity coordinates and reflectance and diffusion spectra for array $\mathrm{r} 5$ on sample A. 

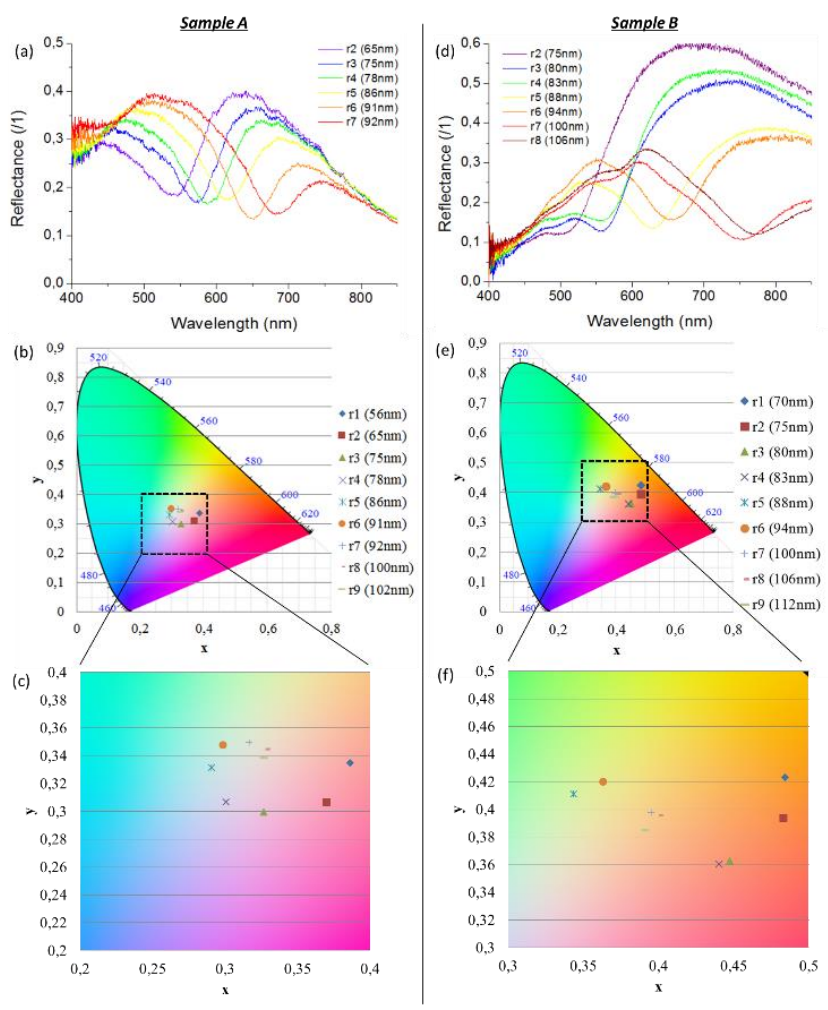

Fig 6 (a,d) Reflectance spectra for selected pixels on samples $A$ and $B$ respectively, (b,e) CIE chromaticity gamut for selected pixels on samples A and B respectively, functioning in reflection, (c) zoom on central region of (b), (f) zoom on central region of (e). The numbers in brackets correspond to the estimated resonator radii in $\mathrm{nm}$.

\section{Physical characterisation results}

The SEM images of arrays r1-r9 on sample A, shown in Fig 7 (a), reveal that the organisation of the islands on a square lattice is maintained for periods at or over 33onm (i.e. r3 and above). As expected, the resonators are seen to increase in size as the array periodicity rises, and the size dispersion within a given array is relatively low leading to the overall homogeneity of the colours observed in the diffusion and reflection measurements. The AFM measurements also enable us to estimate the true aspect ratio of the MRs, which is found to vary between 1 and 1.3 suggesting that the islands are indeed hemispherical to a first approximation. This is further illustrated in Fig 7 (b), showing an AFM image of 9 islands in array r2 on sample $B$. The in-substrate-plane radius of the MRs determined from the SEM images and their heights from the AFM scans are plotted together with the calculated aspect ratio as a function of the array periodicity in Fig 7 (c), where each data point represents a pixel (from r1 to r9).

The structural characterization accounts for the qualitative differences found between the FDTD simulations shown in Fig. 2 (a) and the experimental outcome. As can be seen from the error bars in Fig 7 (c), a dispersion of the islands' dimensions of between $\pm 10 \%$ and $\pm 20 \%$ from the average value is observed. The effect of this dispersion is to "blur" the minima of reflected intensity and so broaden the FWHM of the pass-bands from near $50 \mathrm{~nm}$ in the simulations to approximately 10onm in the measured data, whilst at the same time reducing the depth of the measured pass-band minima.
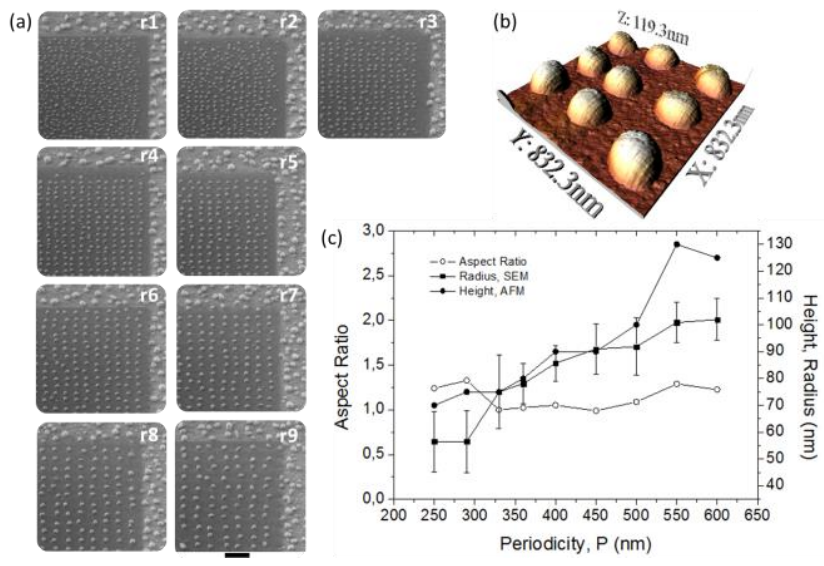

Fig 7 (a) SEM images of the corners of arrays r1-r9 of sample A. Black scale bar $=1 \mu \mathrm{m}$. (b) AFM image of islands in array r2 on sample B. (c) Aspect ratio, in-substrate-plane radius and orthogonal height as a function of lattice periodicity for arrays r1-r9 on sample A.

In spite of the measured size dispersion, the diffusion spectra have similar broadening (FWHM) to those obtained with other techniques. In this case the resonant part of the light can be isolated and the relatively large gap between the islands is not an issue. Another important origin of disorder is the use of amorphous layers leading to less controlled dewetting due to a random formation of grains in the polycrystalline islands and resulting in inhomogeneous distributions of shapes. For the sake of thoroughness we note that for smaller etching periodicities the spatial organization of the dewetted islands is less precise with respect to larger periodicities (e.g. compare r1 with r9 in the SEM images shown in Fig 7 (a)). This fact is mainly ascribed to spurious effects induced by the limited lateral resolution of FIB etching having a larger impact for smaller periodicities. Nonetheless, in spite of a poorer spatial organization of the islands, arrays featuring smaller periodicity exhibit the best performances in terms of spectral selectivity in both diffusion and reflection configurations, thus accounting for the relevance of this approach at shorter wavelengths. Better results could be obtained by using lower milling currents (at the price of a longer milling time) or exploiting better performing etching methods, such as e-beam lithography. Finally, we note that the use of mono-crystalline SOI associated with in situ Ge deposition successfully allows one to limit size, shape and position fluctuations of the islands to within the range of a few percent ${ }^{29}$.

As mentioned earlier, the quality of colour filtering in reflection is lower with respect to diffusion due to the high background on which the resonances are superposed. Apart from size and shape-related issues, the main reason 
for this background is the relatively large spacing of the islands leading to a poor interaction between the impinging light and the MRs. Indeed, in the experimental results we note that the best results in filtering are obtained at smaller periodicities. The easiest way to prevent this problem is to swell the islands further after their formation by supplying additional SiGe after the annealing step in the MBE. Moreover, the use of even thicker initial layers of $\mathrm{SiGe}$ should enable the implementation of the same kind of filters at near-infrared frequencies, also benefiting from reduced absorption losses of SiGe with respect to visible frequencies (not shown).

\section{Conclusion}

We have demonstrated that SiGe alloys are a valuable alternative to pure $\mathrm{Si}$ in manipulating light at the nanoscale with Mie resonators. Reflection and diffusion filters can be formed via dewetting of amorphous layers previously milled with square lattices of varying pitch. The ability to tune the operating wavelength by varying the size of the islands allows for a flexible design that can be adapted to any number of applications. The fabrication technique used allows for the rapid elaboration of large surface area filters and is compatible with existing semiconductor processing techniques due to the relatively low temperature involved. We note that the use of SiGebased alloys in novel electronic devices, such as FET and CMOS transistors, is nowadays extremely important. Because of this, opening the field of dielectric MRs to SiGebased semiconductor alloys is an important step forward towards the integration of photonics with electronic devices.

\section{Methods}

\section{Sample preparation}

The custom SGOI substrates were prepared by thermal oxidation of crystalline $\mathrm{Si}$ (10o) wafers in a rapid thermal processing oven (Jipelec JetFirst). The required oxide thickness of $150 \mathrm{~nm}$ was obtained through a cumulative total of 50 minutes at $1000^{\circ} \mathrm{C}$, split into 5 equal steps to avoid excessive thermal stress on the oven. The oven chamber was first pumped down to approximately 1 Torr before being flushed continuously during the growth process with Oxygen at a rate of $2005 \mathrm{ccm}$. The oxidised wafers were then inserted into the chamber of a molecular beam epitaxy reactor (MBE, Riber), in which the pressure was maintained between $10^{-9}$ and $10^{-10}$ Torr (ultra-high vacuum, UHV) during the deposition of the SiGe alloy. In the case of sample $\mathrm{A}, \mathrm{Si}$ and $\mathrm{Ge}$ were co-deposited at a rate of $16.5 \AA / \mathrm{min}$ and $4.1 \AA / \mathrm{min}$ respectively for 14.5 minutes yielding a $30 n m$ film. For sample $B$, the rates were $14.1 \AA / \mathrm{min}(\mathrm{Si})$ and $3.5 \AA / \mathrm{min}(\mathrm{Ge})$, and the deposition duration 28.4 minutes, yielding a 5onm film. The ratio of the deposition rates (4:1) corresponds to an alloy composition of $\mathrm{Si}_{0.8} \mathrm{Ge}_{0.2}$. The wafers were not heated during the deposition, yielding amorphous films.

The samples were then transferred to an LMIS-FIB milling system (COBRA-type dual beam workstation from Orsay Physics operating at $30 \mathrm{keV}$ ) for templating prior to dewetting. Groups of square lattices approximately $15 \times$ $15 \mu \mathrm{m}$ in size were milled. The milling was performed at a current of $\sim 30 \mathrm{pA}$ using $\mathrm{Si}^{++}$ions selected from an $\mathrm{AuSi}$ source and the total milling time was approximately 30 minutes, corresponding to an average of 30 per $15 \times 15 \mu \mathrm{m}$ lattice.

The following periodicities between the straight line segments making up the lattices were chosen: 250, 290, 330, 36o, 400, 450, 500, 550, 6oonm. This range of periods was used on both samples (A and B), and was chosen in order to yield islands with dimensions comparable to those previously determined in the simulation section by the following reasoning: It is known that for sufficiently small patches (i.e. squares having sides smaller or similar to the characteristic length scale of the underlying dewetting instability), all the mass present in a patch collapses into an individual island (see, for instance, reference [29]). The area of SiGe film required to form a hemispherical resonator of radius $\mathrm{r}$ from a film of thickness $\mathrm{t}$ is $\mathrm{A}=$ $\left(2 \pi r^{3}\right) /(3 t)$. The periodicity $P_{i}$ in this ideal case, where the trench cut by the LMIS-FIB beam is infinitely narrow is therefore $P_{i}=\sqrt{ } A$. However, the effective width (i.e. that providing an equivalent volume of sputtered matter for a trench extending through the entire SiGe layer) of the lines milled by the LMIS-FIB was estimated to be $\sim$ loonm, so the true period required for the lattice is $\mathrm{P}=\mathrm{P}_{\mathrm{i}}+100$. The simulations showed that resonators with radii between 70 and $150 n m$ are required, which correspond to lattice periods between 255 and $585 \mathrm{~nm}$ for a SiGe film depth of $30 n m$ (here referring to sample A). It is arguable that any initial SiGe film thickness could be used, providing the periodicity of the FIB patterning is correctly chosen to yield hemispherical islands of the size wanted. However, in order to avoid spectral features associated with the cut-off of the first diffracted order (Rayleigh phenomenon) that are detrimental to the filters' performances, the lateral period of the islands must be lower than wavelength of operation of each filter array, thus providing a lower limit to the initial film thickness. The thicknesses of the initial SiGe films (3onm for sample A and 5onm for sample B) were chosen to take this constraint into account. Eight groups of lattices (each with $250 \leq \mathrm{P} \leq 600 \mathrm{~nm}$ ) were milled on each sample, with 4 different target thicknesses (each repeated twice): 2, 4, 6, $7 \mathrm{~nm}$. Interestingly, we therefore note that even if the SGOI layer is not completely milled down to the BOX, after annealing a uniform array of isolated MRs can be obtained. This observation is in contrast with the case of monocrystalline SOI, where a non-complete etching leads to a flattening of the milled trenches during annealing, thus losing the islands' organization. This difference may be explained by the 
complex island formation dynamics in amorphous layers associated with re-crystallization during annealing and grain formation ${ }^{41}$.

After ion milling the samples were then returned to the UHV chamber of the MBE reactor for dewetting (annealing). During the transfer process, the samples were immersed in a $4 \%$ concentration aqueous HF solution for 5 seconds in a nitrogen atmosphere, to eliminate any native oxide layer formed on top of the SGOI. The temperature ramping used for the dewetting in ultra-high vacuum was composed of an initial plateau at $500^{\circ} \mathrm{C}$ for 30 minutes to further clean the SGOIs by removing any dirt or organic residues from the surface, followed by a main plateau at $700^{\circ} \mathrm{C}$ for 30 minutes during which the dewetting occurs. Similar results can be obtained at lower temperatures and longer annealing times.

\section{Optical Characterisation}

The samples were characterised by bright- and dark-field microscopy (with a LEICA DMI500oM microscope at 10ox magnification, $\mathrm{NA}=\mathrm{0.75}$ ) in order to observe the reflected and diffused colours, as well as to obtain an indication of the homogeneity of the resonator arrays formed. In the dark-field configuration, the cone of light supplied by the halogen source used to probe the sample is centred at $70^{\circ}$ from the sample surface normal, with collection occurring at all angles allowed by the NA of the objective lens. Following this, diffusion and reflection spectra were collected on a home-made spectroscopy bench, shown in Fig. 3 (b). For reflection measurements, diffused light from a high-power halogen source was focussed into the backfocal-plane of a 10ox Mitutoyo Plan Apo NIR objective $(\mathrm{NA}=0.7)$, giving a near-collimated plane-wave illumination source on the sample surface. For diffusion measurements, light from a secondary fibre-coupled halogen source was focussed onto the sample surface with a lens of focal length $35 \mathrm{~mm}$ at $\sim 70$ degrees from the surface normal. The reflected/diffused light was then collected by the same objective lens used for illumination in the reflectance configuration. The signal was focused onto a multimode optical fibre acting as confocal pinhole and allowing us to select the light reflected/diffused from a circular area with a diameter of approximately $5 \mu \mathrm{m}$ in the sample plane. Thus the spectral investigation is performed over several islands integrating the optical properties over many repetitions of nominally identical MRs. Finally, through the fibre, the light is fed into a spectrometer (Acton IsoPlane SCT-320) equipped with a 150gpmm, $500 n m$-blazed grating and finally imaged onto a Si-CCD array (Princeton Pixis 10o).
Physical Characterisation

Sample A was characterised by atomic force microscopy (AFM) during which $2.5 \times 2.5 \mu \mathrm{m}$ regions of selected resonator arrays were imaged in order to extract the heights of the islands from line profiles following the scan direction. The same arrays were characterised by scanning electron microscopy (SEM) in order to estimate the insubstrate-plane diameter of the islands and examine the array regularity. The sample was oriented at an angle of $45^{\circ}$ relative to the incidence of the electrons, and the lateral field of view was $5.75 \mu \mathrm{m}$. For each array, the top left hand corner was imaged so as to show the contrast between the organisation obtained through templated- and spontaneous-dewetting, as shown in Fig 7. The average substrate-plane radius was estimated through the measurement of 10 islands in the lower left corner of each image via pixel counting.

\section{ASSOCIATED CONTENT}

\section{Supporting Information}

Supporting Information concerns the detail of the FDTD simulations, refractive indices of the SiGe alloy and measurements on the spontaneously dewetted regions. This material is available free of charge via the Internet at http://pubs.acs.org.

\section{AUTHOR INFORMATION}

\section{Corresponding Authors}

* Thomas Wood. E-mail: thomas.wood@im2np.fr

** Marco Abbarchi. E-mail: marco.abbarchi@imznp.fr

\section{Present Addresses}

† INL, Ecole Centrale de Lyon, 36 Avenue Guy de Collongue, 69134 Écully, France.

+ GREMAN, UMR CNRS 7347, Université François Rabelais de Tours, 16 rue Pierre et Marie Curie, 37071 TOURS Cedex 2, France.

\section{Author Contributions}

The manuscript was written through contributions of all authors. All authors have given approval to the final version of the manuscript.

\section{Funding Sources}

We acknowledge the projects PHC MAGHREB (no. 32595SL), EMMAG-Erasmus Mundus Maghreb \& Egypt, the $A^{*}$ MIDEX project (no. ANR-11-IDEX-ooo1-02) funded by the Investissements d'Avenir; a French Government program managed by the French National Research Agency (ANR), the SATT-Sud Est project PROMETHEUS, the Nanotecmat Platform of the IM2NP Institute of Marseille and the $\mathrm{CP}_{2} \mathrm{M}$ microscopy center of AixMarseille University. The Authors thank Rachel V. Zucker and Dominique Chatain for the useful discussions. 
(1) G Mie, Beitrage zur Optik truber Medien speziell kolloidaler Goldlo sungen (Contributions to the optics of diffuse media, especially colloid metal solutions), Ann Phys, 1908, 25, 377-445

(2) Coenen, T., Van De Groep, J., Polman, A., Resonant modes of single silicon nanocavities excited by electron irradiation. ACS Nano, 2013, 7(2), 1689-1698

(3) Van de Groep J., Polman A., Designing dielectric resonators on substrates: Combining magnetic and electric resonances, Opt Exp, 2013, Vol 21, No 22, 26285

(4) Krasnok A, Makarov S, Petrov M, Savelev R, Belov P, Kivshar Y, Towards all-dielectric metamaterials and nanophotonics, Proc. SPIE 9502, Metamaterials X, 2015, 950203

(5) Spinelli P, Verschuuren M A, Polman A, Broadband omnidirectional antireflection coating based on subwavelength surface Mie resonators, Nat Comms, 2012, 3:692

(6) Brongersma M. L., Cui Y., Fan S., Light management for photovoltaics using high-index nanostructures. Nat. Mater. 2014, 13, 451

(7) Jahani S., Jacobs Z., All-dielectric metamaterials, Nat. Nano. 2016, 11, 23

(8) Decker M., Staude I., Resonant dielectric nanostructures : a low- loss platform for functional nanophotonics. Journal of Optics, 2016, 18(10), 1-31.

(9) Spinelli P, Macco B, Verschuuren M A, Kessels W M M, Polman A, A12O3/TiO2 nano-pattern antireflection coating with ultralow surface recombination, Appl. Phys. Lett., 2013, 102, 233902

(10) Dong Fu, Liu J, Song J, Yu H, Zhang Z, Wang W, Xu Y, Song G, Wei X, Coupled optical and electrical study of thin-film InGaAs photodetector integrated with surface InP Mie resonators, Journal of Applied Physics, 2016, 119, 103103

(11) Yue W, Gao S, Lee S-S, Kim E-S, Choi D-Y, Subtractive Color Filters Based on a Silicon-Aluminum Hybrid-Nanodisk Metasurface Enabling Enhanced Color Purity, Scientific Reports, 2016, 6:29756

(12) Proust J, Bedu F, Gallas B, Ozerov I, Bonod N, All-Dielectric Colored Metasurfaces with Silicon Mie Resonators, ACS Nano, 2016, 10 (8), 7761-7767

(13) Zywietz U, Schmidt M K, Evlyukhin A B, Reinhardt C, Aizpurua J, Chichkov B N, Electromagnetic Resonances of Silicon Nanoparticle Dimers in the Visible, ACS Photonics, 2015, 2, 913-920

(14) Van De Groep J, Coenen T, Mann S A, Polman A, Direct imaging of hybridized eigenmodes in coupled silicon nanoparticles, Optica, 2016, Vol. 3, No. 1, 93-99

(15) Staude I, Miroshnichenko A E, Decker M, Fofang N T, Liu S, Gonzales E, Dominguez J, Luk T S, Neshev D N, Brener I, Kivshar Y, Tailoring Directional Scattering through Magnetic and Electric Resonances in Subwavelength Silicon Nanodisks, ACS Nano, 2013, Vol. 7 No. 9, 7824-7832

(16) Evlyukhin A B, Eriksen R L, Cheng W, Beermann J, Reinhardt C, Petrov A, Prorok S, Eich M, Chichkov B N, Bozhevolnyi S I, Optical spectroscopy of single Si nanocylinders with magnetic and electric resonances, Scientific Reports, 2014, $4: 4126$

(17) Coenen T, Van de Groep J, Polman A, Resonant Modes of Single Silicon Nanocavities Excited by Electron Irradiation, ACS Nano, 2013, 7 (2), 1689-1698

(18) Proust J., Bedu F., Chenot S., Soumahoro I., Ozerov I., Gallas B., Abdeddaim R., Bonod N., Chemical Alkaline Etching of Silicon Mie Particles, Adv. Opt. Mater., 2015, Vol 3, Iss 9, 1280

(19) Wang Z. Y., Zhang R. J., Wang S. Y., Lu M., Chen X., Zheng Y. X., Chen L. Y., Ye Z., Wang C. Z., Ho K. M., Broadband optical absorption by tunable Mie resonances in silicon nanocone arrays, Scientific Reports, 2015, 5:7810

(20) Bottein T, Wood T, David T, Claude J B, Favre L, Berbézier I, Ronda A, Abbarchi M, Grosso D, "Black” Titania Coatings Composed of Sol-Gel Imprinted Mie Resonators Arrays, Adv. Funct. Mater., 2017, Volume 27, Issue 2,1604924 
(21) Zywietz U, Evlyukhin AB, Reinhardt C, Chichkov BN, Laser printing of silicon nanoparticles with resonant optical electric and magnetic responses, Nature Communications, 2014, 5:3402

(22) Dmitriev P A, Makarov SV, Milichko VA, Mukhin IS, Gudovskikh AS, Sitnikova AA, Samusev AK, Krasnok AE, Belov PA, Laser fabrication of crystalline silicon nanoresonators from an amorphous film for low-loss all-dielectric nanophotonics, Nanoscale, 2016, 8(9), 5043-8

(23) Kim G. H., Zucker R. V., Ye J., Carter W. C., Thompson C. V., Quantitative analysis of anisotropic edge retraction by solid-state dewetting of thin single crystal films, J. Appl. Phys. 2013, 113, 043512.

(24) Zucker R. V., Kim G. H., Carter W. C., Thompson C. V., A model for solid-state dewetting of a fully-faceted thin film, C. R. Phys. $2013,14,564$

(25) Zucker R. V., Capillary-driven shape evolution in solid-state micro-and nano-scale systems, Ph.D. Thesis, Massachusetts Institute of Technology, 2015

(26) Zucker R. V., Carter W. C., Thompson C. V., Power-law scaling regimes for solid-state dewetting of thin films, Scripta Materialia, 2016, 116, 143-146

(27) Abbarchi M, Naffouti M, Vial B, Benkouider A, Lermusiaux L, Favre L, Ronda A, Bidault S, Berbezier I, Bonod N, Wafer Scale Formation of Monocrystalline Silicon-Based Mie Resonators via Silicon-on-Insulator Dewetting, ACS Nano, 2014, Vol. 8, No. 11, 1118111190.

(28) Naffouti M, David T, Benkouider A, Favre L, Ronda A, Berbezier I, Bidault S, Bonod N, Abbarchi M, Fabrication of poly-crystalline Si-based Mie resonators via amorphous Si on SiO2 dewetting, Nanoscale, 2016, 8, 2844-2849.

(29) Naffouti M, David T, Benkouider A, Favre L, Delobbe A, Ronda A, Berbezier I, Abbarchi M, Templated Solid-State Dewetting of Thin Silicon Films, Small, 2016, 12, 6114.

(30) Albella P., De La Osa R. A., Moreno F., Maier S. A., Electric and magnetic field enhancement with ultralow heat radiation dielectric nanoantennas: considerations for surface-enhanced spectroscopies, ACS Photonics, 2014, 1, 524.

(31) Carletti L., Locatelli A., Neshev D., De Angelis C., Shaping the Radiation Pattern of Second-Harmonic Generation from AlGaAs Dielectric Nanoantennas, ACS Photonics, 2016, 3 (8), 1500-1507

(32) Gutruf P., Zou C., Withayachumnankul W., Bhaskaran M., Sriram S., Fumeaux C., Mechanically Tunable Dielectric Resonator Metasurfaces at Visible Frequencies, ACS Nano, 2016, 133

(33) Khorasaninejad M., Chen W. T., Devlin R. C., Oh J., Zhu A. Y., Capasso F., Metalenses at visible wavelengths: Diffraction-limited focusing and subwavelength resolution imaging, Science 2016, 352, 1190.

(34) Zou C, Gutruf P, Withayachumnankul W, Zou L, Bhaskaran M, Sriram S, Fumeaux C, Nanoscale TiO 2 dielectric resonator absorbers, Optics Letters, 2016, Vol. 41, Iss 15, pp. 3391-3394

(35) Lalanne P, Astilean S, Chavel P, Cambril E, Launois H, Design and fabrication of blazed binary diffractive elements with sampling periods smaller than the structural cutoff, Journal of the Optical Society of America A, 1999, Vol. 16, Issue 5, 1143-1156

(36) Philipp, H. R., E. A. Taft, Optical constants of germanium in the region 1 to $10 \mathrm{eV}$, Physical Review, 1959, 113.4, 1002

(37) Philipp, H. R., E. A. Taft, Optical constants of silicon in the region 1 to $10 \mathrm{eV}$, Physical Review, 1960, 120.1, 37

(38) Naffouti M., David T., Benkouider A., Favre L., Cabie M., Ronda A., Berbezier I., Abbarchi M., Fabrication of core-shell nanostructures via silicon on insulator dewetting and germanium condensation: towards a strain tuning method for SiGe-based heterostructures in a threedimensional geometry, Nanotechnology, 2016, 27, 305602

(39) Celebrano M., Baselli M., Bollani M., Frigerio J., Shehata A.B, Frera A. D., Tosi A., Farina A., Pezzoli F., Osmond J., Wu X., Hecht B., Sordan R., Chrastina D Isella G., Duo L., Finazzi M., Biagioni P., Emission engineering in germanium nanoresonators, ACS Photonics, $2012(1), 53-59$

(40) Gómez-Medina R., García-Cámara B., Suárez-Lacalle I., González, Moreno F., Nieto-Vesperinas M., Sáenz J. J., Electric and magnetic dipolar response of germanium nanospheres: interference effects, scattering anisotropy, and optical forces, J. Nanophoton., 2011, 5, 053512

(41) Wakayama, Y., Tagami, T., Shun-Ichiro. T., Formation of Si islands from amorphous thin films upon thermal annealing. Journal of Applied Physics, 1999, 85(12), 8492 
(42) Korzec, M. D., Wagner, B., Roczen, M., Rech, B., Schade, M., Equilibrium shapes of poly-crystalline silicon nanodots, Journal of Applied Physics, 2014, 115, 074304

(43) Wakayama, Y., Tagami, T., Shun-Ichiro. T., Three-dimensional islands of Si and Ge formed on SiO2 through crystallization and agglomeration from amorphous thin films. Thin Solid Films, 1999, 350(1), 300-307.

(44) Humlíček, J., Garriga, M., Alonso, M. I., Cardona, M., Optical spectra of SixGe1-x alloys. Journal of applied physics, 1989, 65(7), $2827-2832$

(45) Ahuja, R., Persson, C., Da Silva, A. F., De Almeida, J. S., Araujo, C. M., Johansson, B., Optical properties of SiGe alloys. Journal of Applied Physics, 2003, 93(7), 3832-3836.

(46) Bahng, J. H., Kim, K. J., Ihm, S. H., Kim, J. Y., Park, H. L., Evolution of optical constants and electronic structure of disordered Si1xGex alloys. Journal of Physics: Condensed Matter, 2001, 13(4), 777.

(47) Sutter E., Sutter P., Assembly of Ge nanocrystals on SiO2 via a stress-induced dewetting process, Nanotechnology, 2006, 17, 3724

(48) Zhang P, Yang B, Rugheimer P, Roberts M, Savage D, Liu F Lagally M, Influence of germanium on thermal dewetting and agglomeration of the silicon template layer in thin silicon-on-insulator, J. Phys. D: Appl. Phys. 2009, 42, 175309

(49) Vynck K., Burresi M., Riboli F., Wiersma D. S., Photon management in two-dimensional disordered media, Nat. Mater. $2012,11,1017$.

(50) Van Lare M.-C., Polman A., Optimized Scattering Power Spectral Density of Photovoltaic Light-Trapping Patterns, ACS Photonics $2015,2,822$

(51) Sapienza R., Thyrrestrup H., Stobbe S., Garcia P. D., Smolka S., Lodahl P., Cavity Quantum Electrodynamics with Anderson-Localized Modes, Science 2010, 327, 1352.

(52) Strudley T., Zehender T., Blejean C., Bakkers E. P., Muskens O. L., Mesoscopic light transport by very strong collective multiple scattering in nanowire mats, Nat. Photonics 2013, 7, 413.

(53) Wiersma D. S., Disordered photonics, Nat. Photonics 2013, 7, 188

(54) Riboli F., Caselli N., Vignolini S., Intonti F., Vynck K., Barthelemy P., Gerardino A., Balet L., Li L. H., Fiore A., Gurioli M., Wiersma D. S., Engineering of light confinement in strongly scattering disordered media, Nat. Mater. 2014, 13 (7), 720.

(55) Kuznetsov A I., Miroshnichenko A. E., Fu Y. H., Zhang J., Luk’yanchuk B., Magnetic light, Sci. Rep. $2012,2,492$. 